\title{
Self-Optimization of Pilot Power in Enterprise Femtocells Using Multi objective Heuristic
}

\author{
Lina S. Mohjazi, ${ }^{1}$ Mahmoud A. Al-Qutayri, ${ }^{1}$ Hassan R. Barada, ${ }^{1}$ \\ Kin F. Poon, ${ }^{2}$ and Raed M. Shubair ${ }^{1}$ \\ ${ }^{1}$ College of Engineering, Khalifa University of Science, Technology and Research, UAE \\ ${ }^{2}$ Etisalat-BT Innovation Centre (EBTIC), UAE \\ Correspondence should be addressed to Lina S. Mohjazi, lina.mohjazi@kustar.ac.ae
}

Received 30 March 2011; Accepted 17 October 2011

Academic Editor: Youyun Xu

Copyright (C) 2012 Lina S. Mohjazi et al. This is an open access article distributed under the Creative Commons Attribution License, which permits unrestricted use, distribution, and reproduction in any medium, provided the original work is properly cited.

\begin{abstract}
Deployment of a large number of femtocells to jointly provide coverage in an enterprise environment raises critical challenges especially in future self-organizing networks which rely on plug-and-play techniques for configuration. This paper proposes a multi-objective heuristic based on a genetic algorithm for a centralized self-optimizing network containing a group of UMTS femtocells. In order to optimize the network coverage in terms of handled load, coverage gaps, and overlaps, the algorithm provides a dynamic update of the downlink pilot powers of the deployed femtocells. The results demonstrate that the algorithm can effectively optimize the coverage based on the current statistics of the global traffic distribution and the levels of interference between neighboring femtocells. The algorithm was also compared with the fixed pilot power scheme. The results show over fifty percent reduction in pilot power pollution and a significant enhancement in network performance. Finally, for a given traffic distribution, the solution quality and the efficiency of the described algorithm were evaluated by comparing the results generated by an exhaustive search with the same pilot power configuration.
\end{abstract}

\section{Introduction}

Coverage and capacity are two major aspects which operators have to address while offering new mobile multimedia services to their customers such as video on demand, web 2.0 services, and social networking. At the same time, indoor coverage presents many challenges in the current $3 \mathrm{rd}$ generation (3G) (e.g., UMTS) and future 4th generation (4G) (e.g., LTE) cellular networks. Those networks operate at higher frequencies in comparison with the conventional 2nd generation (2G) (e.g., GSM) networks [1]. Consequently, signal penetration through building walls becomes a complex process. This fact creates a real challenge, especially that studies on wireless usage show that more than $50 \%$ of voice calls and more than $70 \%$ of data traffic are generated indoors [2]. Therefore, proposing data intensive services in conjunction with the presence of the indoor coverage challenges are the main drives for deploying specific devices such as femtocells to complement the traditional outdoor base stations.

A femtocell is a short-range (up to $40 \mathrm{~m}$ ) low-cost lowpower base station (BS) installed by end users indoors to enhance voice and data receptions. Femtocells make use of the broadband connections such as digital subscriber line (DSL), cable modem, or a separate radio frequency (RF) backhaul channel to communicate with the cellular network [3] as shown in Figure 1.

Unlike other wireless indoor solutions, such as relays and picocells, femtocells are connected to the cellular operator via internet. Therefore, they do not need to be planned carefully and maintained by cellular operators. Both capital expenditures (CAPEXs) and operational expenditures (OPEXs) are expected to be remarkably lowered and therefore, femtocells will increasingly attain a strong appeal by operators. According to the market analysis carried out in [4], about 23 million femtocell devices are expected to be sold worldwide within 


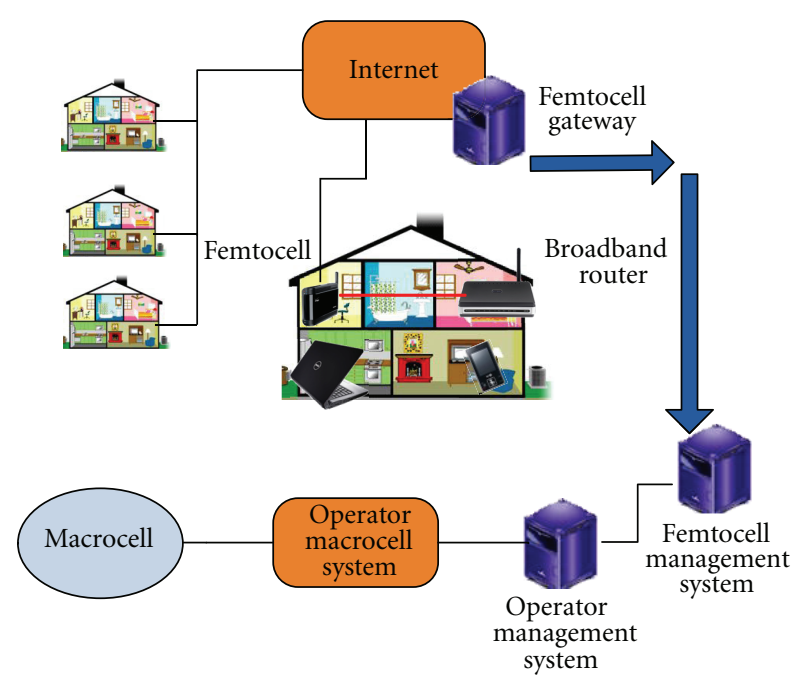

Figure 1: Basic femtocell network.

the next few years for a total market of over one billion dollars.

The major benefits of femtocells to both operators and end users are summarized below.

(a) Femtocell benefits for operators [1] are as follows.

(i) Increased network capacity: indoor traffic will be offloaded to femtocells and operators will relieve stress on macrocell networks which in turn increase the network capacity.

(ii) Lower capital costs: deploying femtocells will reduce the cost required on extending the macrocell layer as the number of subscribers in a mobile operator's network increases.

(iii) Expanded revenue opportunities: the average revenue per user (ARPU) will be raised due to the increase in using mobile multimedia services when the provided coverage is excellent and superior broadband wireless performance is available.

(iv) Lower backhaul costs: the cost of backhauling traffic to the operator's core network will not be handled by the operator anymore. Instead, it will be handled by the user via DSL, cable, or fiber access lines.

(v) Increased customer stickiness and conversion: more users will be attracted to the operators family plans when they experience high quality in-home coverage and home zone calling plans

(b) Femtocell benefits for end users [5] are as follows.

(i) Better coverage: users will experience better signal quality and higher communication reliabilities and throughputs because they operate on short transmit-receive distance

(ii) Faster access: due to the availability of high performance mobile data, faster access to mobile services and multimedia content will be available.

(iii) Easier bill tracking: femtocells enable a converged billing infrastructure allowing a subscriber to receive a single bill providing itemized information on all communications services consumed by a subscriber. Subscribers also benefit from unlimited calling instead of perminute charges.

(iv) Prolonged mobile battery life: mobile terminals transmit at significantly less powers due to the short distance separation from the femtocell.

Femtocells can be deployed in three different scenarios; home, enterprise, and hotspots. According to Informa Telecoms \& Media, the number of femtocell customer premises equipment vendors is expanding at an accelerated base [6]. These vendors are also starting to expand their product lines to enterprise and larger area femtocells.

This paper focuses on enterprise environment where femtocells are deployed in companies and thus, no concrete planning is needed and only rudimentary settings are required. However, in contrast with the residential deployment where interference between femtocells is not a major concern, the interference between femtocells in enterprise environment has to be eliminated. As a result, when dense femtocell deployment takes place to jointly provide seamless coverage in a large area, it forms a real challenge. Therefore, self-organizing network (SON) capabilities have to be embedded in the physical device of a femtocell for instant downlink power control. Adaptive downlink power control is required to reduce the overlaps between cells while maintaining a good coverage at the same time depending on the load and locations of the users [7].

Another important aspect that is stated in [8] and needed to be addressed in the SON capabilities of a femtocell is load balancing. The arrival of mobile users and the resulting traffic generated by the users are random, time varying, and often unbalanced. This eventually leads the cells in the network to handle unequal amounts of load. In this situation, some cells will be overloaded and the resources of others might not be fully utilized.

The load balancing problem becomes even more crucial when Long Term Evolution (LTE) networks are deployed. This could be anticipated by the following two situations. First, due to the rapid development of network applications and services, the resources of a particular femtocell will soon be running out if they are not well balanced. Second, traffic patterns are time varying and unpredictable. In that event, the network has to dynamically adapt its resources in a timely fashion according to the varying load. Obviously, this could not be achieved by static and prefixed network planning. Therefore, SON capabilities are essential in the femtocell operation to address a large-scale femtocell deployment. In order to successfully execute the process of self-organization, three main functions have to be performed as described in [9]: self-configuration, self-optimization, and self-healing. Figure 2 illustrates the life cycle of a self-organizing network. 


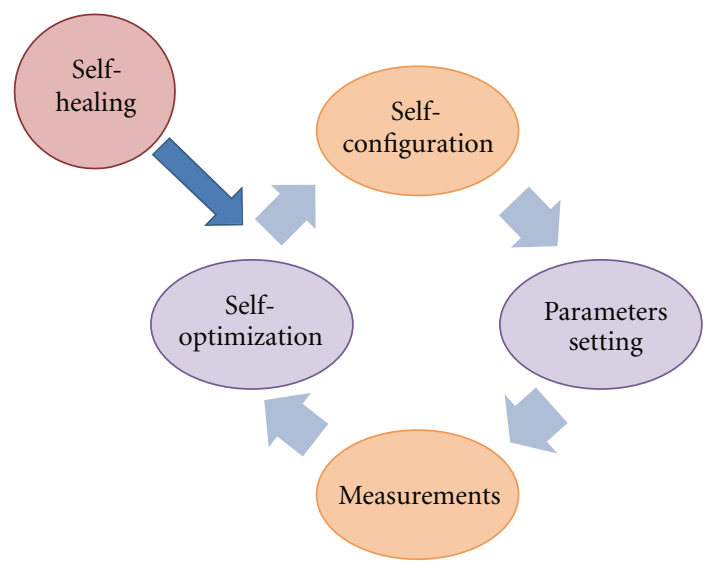

FIgURE 2: Life cycle of a self-organizing network.

The three processes of a self-organizing network are described as follows.

(a) Self-configuration: this process is a preoperational state and is done each time a new femtocell is deployed in the network. It has to configure its initial settings including IP address, association with a gate way, neighbor list, radiated power, channels, and handover parameters.

(b) Self-optimization: in this process, with the help of an external optimization tool, the femtocell tunes its parameters to optimize the network according to the varying conditions of the surrounding environment such as traffic demands and users locations. This process takes place in the operational state and depends on measurements taken from user equipment (UE) as well as from femtocells.

(c) Self-healing: in contrast with microcells and picocells, femtocells are not maintained by the operator. Therefore, femtocells should be able to automatically detect and localize then heal failures of the network such as reducing the output power in case of temperature failure.

As described in the SOCRATES (Self-Optimisation and self-ConfiguRATion in wirelEss networks project) framework for future wireless networks [10], SON can be implemented in three different approaches: distributed, centralized, and hybrid.

(a) Distributed SON: the functionalities of an $S O N$ reside in each evolved NodeB (eNB) and self-optimization algorithms are executed on a local basis inside the eNB itself.

(b) Centralized SON: all optimization algorithms are executed only in a central node. In most cases, it is performed in the Operations, Administrations, and Maintenance (OAM) system. Individual eNBs have no role in carrying out independent actions.

(c) Hybrid SON: depending on the network requirements, self-organization tasks are divided between individual eNBs and a central node.
In a distributed SON, the adjustment of parameters will have a local scope and is difficult to support complex optimization schemes. They generally require the coordination of numerous eNBs whereas a centralized SON has the ability to control the parameters of an entire network. Furthermore, the centralized SON could be best fit in situations where there is a need to manage the interaction between different cells.

Accordingly, the optimization algorithm proposed in this paper is dedicated to the centralized approach. The objective is to centrally and dynamically optimize the coverage of a group of femtocell base station (FBSs) based on a multi objective function. The algorithm simultaneously minimizes the interference, reduces the coverage gaps, and balances the load of all FBSs in the network deployed in an enterprise scenario.

The remainder of the paper is organized as follows: related work is presented in Section 2. Section 3 defines the problem statement and addresses the proposed system architecture. The application of the GA to the selfoptimization of enterprise femtocell pilot powers is described in Section 4. Section 5 presents the simulation parameters and the results achieved. Finally, the conclusions and future work are presented in Section 6.

\section{Related Work}

Most of the published literature concentrate on optimizing the locations of base stations to achieve certain requirements such as coverage and capacity targets, minimizing average bit error rate (BER), and so forth, as in [11-15]. However, although this kind of optimization is applicable to theoretical networks, it has some limitations in practical networks where several aspects restrict the operation of base stations including zoning and power emissions. Therefore, since the introduction of new and complex cellular technologies, the recent trend in research is converging towards employing optimization techniques that assume fixed base station locations and optimize their settings instead. Those settings basically characterize the network performance and usually include pilot channel power, antenna tilt, and azimuth. This can be evidenced by the research carried out by Siomina et al. [16] and Ho et al. in [17].

As discussed in the previous section, dynamic optimization of the femtocell parameters is essential for its successful deployment in current and future cellular networks. One of those parameters is Common Pilot Channel (CPICH) as in Universal Mobile Telecommunications System (UMTS) and High Speed Downlink Packet Access (HSDPA) networks. The CPICH power is usually set to a fixed value between 10 and 20 percent of maximum transmit power in conventional macrocell networks. The CPICH signal is used by mobile terminals to estimate channel quality, cell selection/reselection, and handover evaluation. CPICH power also determines the cell coverage. In other words, higher $\mathrm{CPICH}$ power leads to larger cell coverage area. The maximum downlink transmit power of a base station is constant, and hence, the amount of CPICH affects the cell performance. Lower 
$\mathrm{CPICH}$ power means more power is left for traffic and cell overlaps are reduced. However, higher $\mathrm{CPICH}$ power increases the downlink interference due to the increased cell overlaps. As a result, a tradeoff has to be done between coverage and pilot pollution when implementing pilot power adaptation schemes.

A number of studies investigated the effect of pilot power in scenarios where femtocells are deployed. The work in [18] proposed a power control scheme to uniformly configure a constant 10-meter cell radius. The main purpose of the study was to reduce the interference levels of residential femtocells to the macrocell users. In addition, the work done in [19] demonstrated that when femtocells are deployed, the call dropping probability experienced by macrocell users is potentially minimized when power adaptation techniques are implemented. Several studies in the literature address the topic of self-organization of home femtocells [20-22] where femtocells are in the presence of neighboring macrocells and femtocells.

In the literature to date, only a few studies deal with self-organization of enterprise or a group of femtocells. The aim of coverage optimization differs amongst the network requirements and constraints of the three femtocell types. For example, in a home femtocell deployment, the key objective of coverage optimization is to minimize leakage of coverage by a single femtocell to public areas as in $[19,23]$.

On the contrary, when a number of femtocells are densely deployed such as in an enterprise environment, the aim would be to jointly provide seamless coverage throughout the whole area. Previous work related to optimizing the downlink pilot power of a group of femtocells through an autonomous central control unit mainly focuses on maximizing the cell coverage while mitigating the interference between user-deployed femtocells [24, 25]. In [26] Zhang et al. address power management. They present an algorithm based on linear programming to minimize pilot power in dense residential HSDPA femtocells deployment. On the other hand, the research carried out in [27, 28] proposes a distributed radio coverage optimization in enterprise femtocells. In [29], Wei et al. use the Voronoi diagram to control the coverage area of each femtocell in a public area.

\section{Proposed Centralized Self-Optimization of Pilot Power}

This paper expands on the work highlighted in [3]. The objective of the proposed algorithm is to centrally and dynamically optimize the coverage of a group of femtocell base station (FBSs) based on a multi objective function that would simultaneously minimize the interference, reduce the coverage gaps, and balance the load of all FBSs in the network. This paper presents the implementation of a heuristic approach based on the genetic algorithm (GA) for coverage optimization in an enterprise femtocell network.

Due to the plug-and-play manner by which femtocells are deployed, autonomous power control is required to achieve the objectives described in Section 3.2 below. In order to achieve that, the proposed algorithm is run by the OAM

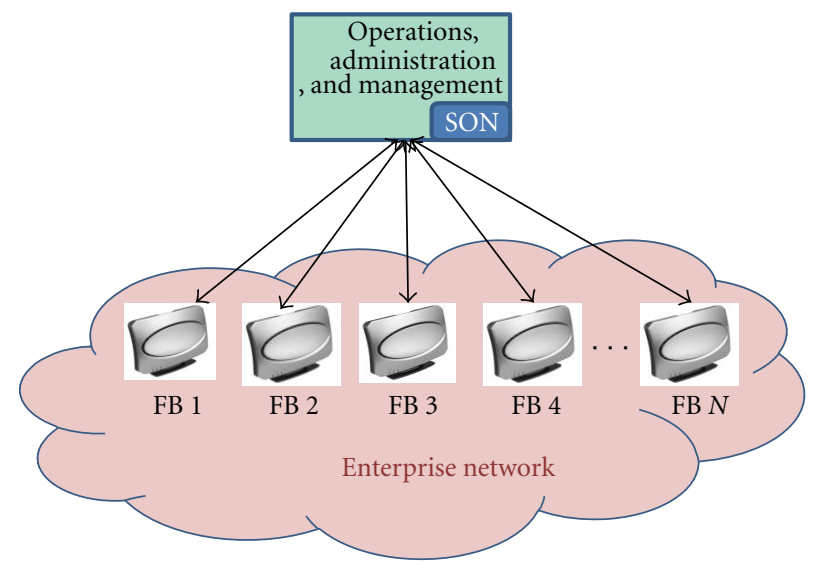

FIGURE 3: Centralized self-optimizing enterprise femtocell network.

after collecting the required statistics from the femtocells through the broadband link such as DSL, TV Cable, or others. As a result, the downlink pilot power of the group of femtocells is adaptively changing according to the global traffic distribution in the network. Our optimization model autonomously finds the best pilot power configuration for $\mathrm{N}$ user-deployed femtocells provided that the OAM is aware of local knowledge available for all femtocells. The effectiveness of the proposed approach was evaluated by simulating a real enterprise environment that incorporates a comprehensive indoor channel model.

3.1. System Architecture. This section provides an overview of the centralized self-optimizing cellular network. Figure 3 shows the basic elements that make up the architecture of such a network.

The network operator deploys the macrocellular infrastructure to provide endusers with public telephone and lowrate data access. Within this infrastructure, the femtocells are deployed by endusers in an enterprise in an unstructured fashion. Although the distribution of these small cells could be significantly irregular in geography, the network operator still has to manage the whole cellular interference by employing the OAM. The proposed optimizer is supposed to be executed within the OAM after receiving the required measurement reports from all FBSs deployed in the network. Depending on those measurements, the algorithm compares the current network behavior with the desired one, then determines the optimal or near optimal pilot power configurations of the FBSs. At the end of the process, the OAM sends the new pilot power settings to the connected FBSs. This process resembles a centralized SON as described in Section 1. The block diagram of the process is illustrated in Figure 4.

The following is a general description of the major components for an enterprise network deploying $N$ femtocells.

(1) Small base station/femtocell: a small device that is solely deployed and maintained by the user in a plugand-play manner. Interference management is fully optimized by the OAM. 


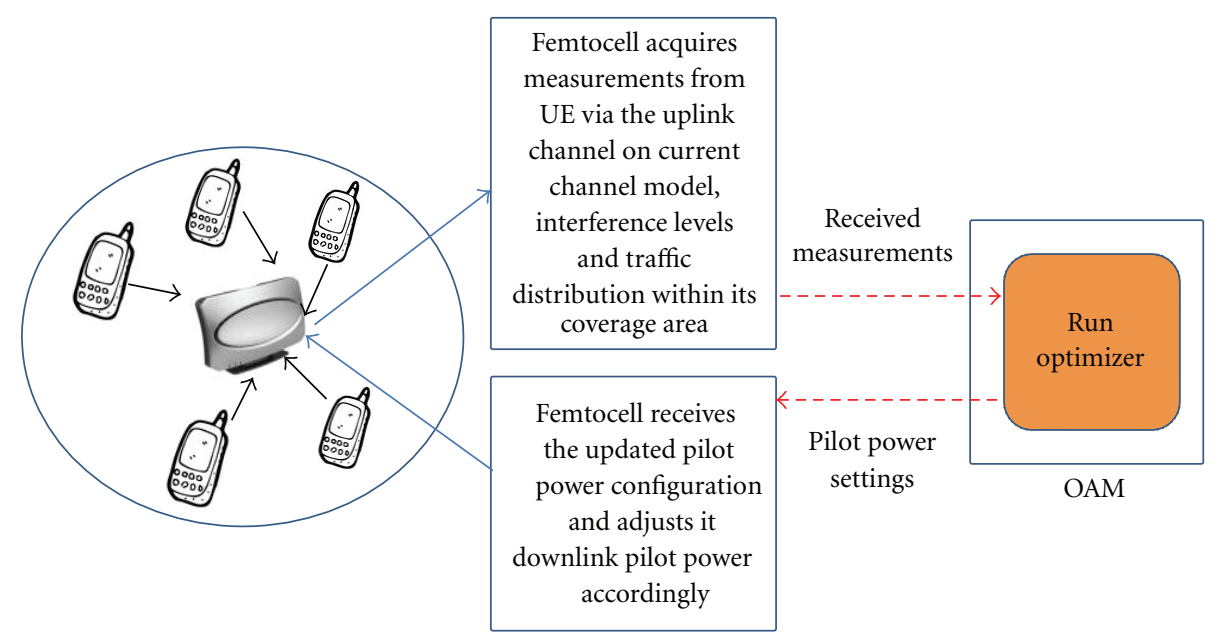

FIgURE 4: Self-optimization of pilot power diagram.

(2) Operations, Administration, and Management (OAM): a smart part of the architecture introduced for autonomous spectrum management and dynamic resource allocation through SON capabilities. When traffic distribution changes, the OAM can adaptively configure the femtocell's radio parameters and optimize its resource allocation through executing SON functionalities.

(3) User Equipment (UE): a mobile device that has spectrum sensing and access capabilities. Major features of future UE terminals will more likely incorporate cognitive radio- and software-defined radio capabilities.

3.2. Problem Description. The objective is to provide endusers with cellular services in an intended area, such as a large enterprise. Therefore, a group of $N$ femtocells are deployed without careful cell planning to jointly achieve this requirement. The coverage area of a FB is the region where its downlink transmitted pilot signal is the strongest signal received by mobile terminals and its value is greater than a predefined threshold $\varepsilon$. Therefore, the number of terminals connected to a certain FB depends on the received pilot power received from that $\mathrm{FB}$. In the presented optimization, the role of the OAM is to adjust the pilot power and thus the coverage of the femtocells in order to fulfill the following objectives.

(i) Minimize the coverage overlaps between neighboring femtocells. The objective is to reduce the interference levels caused by adjacent femtocells as much as possible. In addition, minimize pilot pollution level.

(ii) Minimize the coverage gaps within the specified area where femtocells are to be deployed. By doing this, the total coverage area is maximized and more traffic is offloaded from the outdoor macrocell. Moreover, the amount of signaling due to the femto-macro or macro-femto handovers is substantially reduced. (iii) Balance the load handled amongst all femtocells in the covered area. The aim here is to abstain overloading or underutilization which affect the call dropping/blocking probability.

Depending on the operator's requirements, weighting factors are introduced in order to emphasize on different objectives as some of them conflict with each other. For example, minimizing the overlaps would subsequently increase the coverage, which may cause some of the femtocells to be underutilized.

\section{GA Optimization Model for Femtocell Pilot Power Adjustment}

The purpose of formulating the optimization problem is to find the optimal or near-optimal downlink pilot power configuration of $N$ deployed femtocells to fulfill the three previously presented requirements. Since multiple solutions (i.e., multiple $\mathrm{CPICH}$ configurations) can be considered as candidate solutions to this particular problem, GA has the potential advantage as a multipoint search engine for optimization problems with multiple objectives. The number of possible CPICH configurations is massive, and this number grows as the number of femtocells in the network increases. As a result, it would be almost impossible to find an optimum solution within a reasonable amount of computing time without employing any optimization techniques, specifically when having to deal with several variables and constraints. In order to perform such optimization, the radio propagation characteristics of a given area need to be included in the objective function. Another issue in the design is the existence of conflicting objectives. For example, increasing the $\mathrm{CPICH}$ of a FB would increase the coverage, but doing so might also increase the load that the FB is handling, and eventually cause an increase in the overlaps with neighboring FBSs. This kind of problem does not have a closed form solution and therefore, is a nonconvex optimization problem with numerous local optima. Moreover, it can be shown that 
it is an NP-hard and could not be solved in polynomial time. The only way to find a global optimal solution is through a computationally intensive, exhaustive analysis, in which all possible inputs are tested. However, it can only be achievable for a very small number of femtocells. Therefore, a heuristic technique is applied to solve such a problem. Heuristics represent a family of approximate optimization techniques that have gained a lot of popularity over the years. They are considered to be the most promising and successful techniques. Moreover, they provide acceptable solutions in a reasonable time for solving hard and complex problems in science and engineering [30]. One of the most wellknown and commonly used heuristics is GA. GA belongs to the evolutionary algorithm family that mimics the process of natural evolution. This algorithm has several advantages over other optimization techniques including parallelism, robustness to dynamic changes, and can be hybridized with other methods.

Given the above stated advantages, GA has been chosen to be applied in this research in order to adjust the coverage of a group of femtocells periodically. In addition, GA has proven to have a fast convergence time [31], and that is necessary for the dynamic environment where the femtocell is to be deployed. It is assumed that the GA is periodically executed by the OAM embedded processor to modify the pilot power of the femtocells after performing the process of data acquisition from the network.

4.1. Overview of Genetic Algorithm. GA is a method that uses genetics as its archetype for problem solving. GA is based on the survival of the best individual in a population [31]. Each individual or solution represents a chromosome in a population. Based on a specific fitness function, all individuals undergo a test to measure their fitness. For each population and depending on the selection rate and method, individuals having the highest fitness are more likely to be selected as parents to go through the reproduction process. The effect of reproduction is very important as it produces children that inherit a combination of good characteristics from two different parents. Finally, mutation is done for the children before including them in the new population.

The following steps should be performed to solve a problem using GA.

(1) Fitness evaluation function: a fitness or an objective function is defined according to the parameters and constraints of the problem to be solved.

(2) Encoding style: the way a solution to be represented as a chromosome in the algorithm is selected through the different encoding styles that exist (binary, octal, permutation, value, etc.)

(3) Initialization: the first population is generated where each bit of the chromosome is randomly generated depending on the encoding style.

(4) Evaluation: the fitness of each chromosome is calculated according to the objective function.

(5) Selection: this step is done randomly with a probability depending on the relative fitness of the

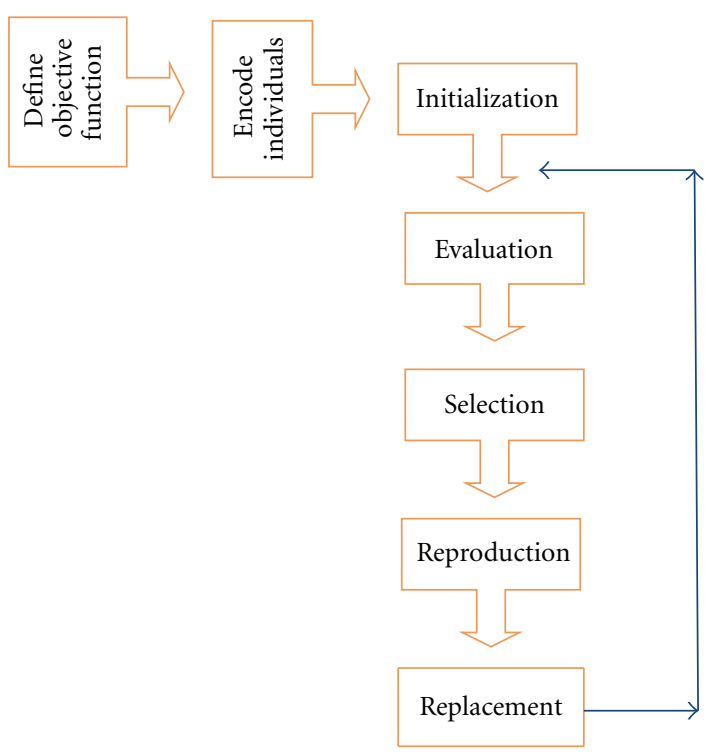

Figure 5: Process of a genetic algorithm.

chromosome; the higher the fitness, the higher the chance of being chosen for reproduction.

(6) Reproduction: genetic operators such as crossover and mutation on selected chromosomes are executed to produce children.

(7) Replacement: this is the last step where chromosomes from the old population are replaced by the generated children.

(8) Steps 4 through 7 are repeated until a predefined number of iterations is reached or no further improvement can be observed within a defined period. Figure 5 demonstrates an overview of the GA process described above.

\subsection{GA Setup for Coverage Optimization}

4.2.1. Presentation of Population. In order to formulate the problem, it was assumed that $N$ user-deployed FBSs are connected to a centralized self-optimizing management system such as the OAM. The locations of the FBSs are fixed by the enterprise owner with no precise cell planning but with some elementary arrangements. Therefore, it is intended to find a vector $\mathbf{P}=\left(\mathcal{P}_{1}, \mathcal{P}_{2}, \mathcal{P}_{3}, \ldots, \mathcal{P}_{N}\right)$ where each element in the vector denotes the downlink pilot power strength for a specific FB $i$ such that $i \in N$. This is done by the OAM on a continuous basis. This means that the OAM periodically collects measurements from all FBSs in the network about the surrounding environment (i.e., traffic distribution, power levels received at mobile users, etc.) and then sends the updated pilot power level to each FB accordingly.

Therefore, each vector $\mathbf{P}$ is considered as a possible solution in the population and affects the three objectives 
defined in the objective function. Population $\mathbf{K}$ is represented as follows:

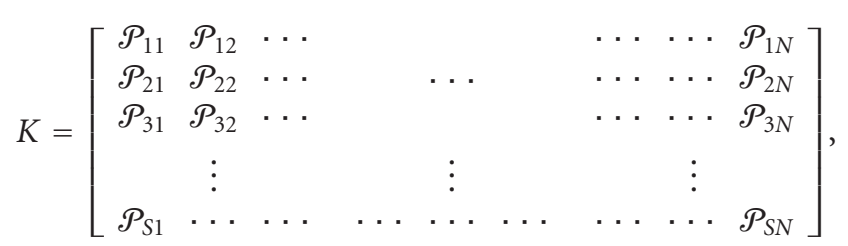

where $S$ is the population size. The pilot power of each cell is randomly generated. The elements of $\mathbf{P}$ are constrained such that min power $<\mathcal{P}_{s i}[m W]<$ max power, where $s \in S$. Consequently, the value encoding style was used to enumerate the possible solution into chromosomes.

4.2.2. Objective Function. The algorithm presented adjusts the FB's pilot power channel and eventually its coverage area depending on the collected measurements and statistics from the $N$ FBSs in the network. The objective function used in this paper is similar to the one utilized in the research carried out in [28]. However, in this method, fitness is centrally computed and is based on the global knowledge of the network conditions.

Formulation of the objective function that takes into account the three objectives described in Section 3.2 is one of the most important tasks to be performed in order to evaluate the fitness of each individual in the population K. Several methods such as finding a Pareto-optimal set, weighted sum, or hierarchical optimization are often applied to solve a multi objective problem. The weighted sum is the most widely used approach and is employed in this research. This method allows the scores of all objectives to be summed up into an aggregate fitness value. This is done by multiplying each objective function by a weighting factor and summing up all weighted objective functions. Therefore, when GA is applied to this 3-objective optimization problem, the values of the three objective functions for each individual have to be estimated. Then, according to these values the overall fitness value of each individual could be calculated. As a consequence, GA searches for an individual $(s)$ with a better fitness as in the normal case of a single-objective optimization problem. In order to transform the values of the three objectives into one single value, the three objectives are combined to produce one scalar function.

The three objective functions used to formulate the main objective function are described below. Three types of statistics are required from each $\mathrm{FB}$ in order to process the GA.

(1) The first function $f_{L}(s)$ is used to maximize the load experienced by all FBS in the network. The load that a femtocell can handle at any time, $L_{i}$, is subject to an upper bound $L_{\text {th }}$ : the purpose here is to balance the load handled by the FBSs across the network and block overloading. Therefore, each femtocell reports back to the OAM the load of voice traffic in Erlangs that it is handling. In order to capture the load of the whole network, the mean value of all FBSs load is calculated as follows:

$$
f_{L}(s)= \begin{cases}\frac{1}{N} \sum_{i=1}^{N} \frac{L_{i}}{L_{\mathrm{th}}} & \text { if } L_{i} \leq L_{\mathrm{th}} \\ 0, & \text { otherwise }\end{cases}
$$

here a femtocell $i$ estimates the load that it is handling $L_{i}$ for all its covered users $U$ by

$$
L_{i}=\sum_{j=1}^{U} L_{j} \quad \text { if } P r_{j i}=P t_{s i}-P L_{i j} \geq \varepsilon,
$$

where $P r_{j i}$ is power received by user terminal $j$ from $\mathrm{FB} i, P t_{s i}$ is $\mathrm{CPICH}$ transmitted from $\mathrm{FB} i$ in individual $s$, and $P L_{i j}$ is path loss between $\mathrm{FB} i$ and user terminal $j$.

It can be observed that whenever the load of femtocell $i$ does not exceed $L_{\text {th }}$, the value of $f_{L}$ increases. This allows the femtocells to take more load as long as their load is less than $L_{\text {th }}$. The path loss $P L(\mathrm{~dB})$ model used to approximate the path loss between a FB and a mobile terminal is

$$
P L(\mathrm{~dB})=38.5+\eta \times 10 \log _{10}(d)+\sum_{f=1}^{F} a_{f} W_{f},
$$

where $\eta$ is the distance power decay factor, $d$ is the direct transmitter-receiver distance in meters, $\alpha_{f}$ is the number of penetrated walls of type $f, W_{f}$ is the attenuation due to the wall of type $f, f=1,2, \ldots, F$. In our simulations, $\eta=2$. Moreover, correlated shadow fading with a standard deviation of $8 \mathrm{~dB}$ was considered to reflect the effect of obstacles such as furniture.

(2) The second objective function $f_{G}(s)$ is used to minimize the probability of users in coverage gaps. A coverage gap is part of the total area intended to be covered where a mobile terminal does not receive any pilot power from any of the FBSs that is above $\varepsilon$. This is calculated by each FB $i$ and can be achieved by measuring the number of handovers that occurred between the FB $i$ and the underlay macrocell. The probability of users entering a femtocell coverage gap $G_{i}$ should not exceed an upper bound $G_{\text {th }}$. The mean value of the coverage gaps of the whole network is computed using

$$
f_{G}(s)=\frac{1}{N} \sum_{i=1}^{N} G_{i}
$$

where

$$
G_{i}=\frac{u_{G}}{u_{G}+u_{i}},
$$

where $u_{G}$ is the number of users handed over from femtocell $i$ to the macrocell, and $u_{i}$ is the number of users handed over from femtocell $i$ to a neighboring femtocell. 
(3) The third objective function $f_{V}(s)$ is employed to minimize the coverage overlaps in the network: an overlap $V_{i}$ represents a fraction of the coverage area of a FB $i$ intersecting with the coverage area of neighboring femtocells. In order to perform the process of overlap estimation as in (7), a femtocell $i$ keeps record of the received measurement reports gathered from mobile terminals $u_{V}$ where more than one pilot power measured by a mobile terminal is higher than $\varepsilon$. Therefore, $V_{i}$ is calculated as the ratio of $u_{V}$ to the total number of pilot power measurements sent back by all users, $U$, in FB $i$. The algorithm restricts $V_{i}$ from surpassing an upper bound $V_{\text {th }}$. Then $f_{V}(s)$ for the whole network is approximated as in (8)

$$
\begin{gathered}
V_{i}=\frac{u_{V}}{U}, \\
f_{V}(s)=\frac{1}{N} \sum_{i=1}^{N} V_{i},
\end{gathered}
$$

where

$$
u_{V}=\sum_{b=1}^{B} \sum_{j=1}^{U} u_{b j}
$$

where $B$ is the number of neighboring femtocells of femtocell $i$. Here, $u_{b i}$ is the number of users who's both $P r_{j i}$ and at least one $P r_{j b}$ are greater than $\varepsilon$.

The GA is executed in the OAM to maximize the overall objective function $f(s)$ defined in (10). In this case, evaluation of how good the whole network performance is would be performed for each individual $(s)$ in the population K.

$$
f(s)=\frac{\omega_{L} \cdot f_{L}(s)+\omega_{G} \cdot\left(1-f_{G}(s)\right)+\omega_{V} \cdot\left(1-f_{V}(s)\right)}{\omega_{L}+\omega_{G}+\omega_{V}},
$$

where $f_{L}(s), f_{G}(s)$, and $f_{V}(s)$ are normalized between 0 and 1. Moreover, as stated earlier, depending on the requirements of the operator more emphasis can be put on one objective over the others through the weighting factors $\omega_{L}, \omega_{V}$, and $\omega_{G}$ which can take values between 0 and 1 .

According to the previous mathematical formulation, $L_{\text {th }}, G_{\text {th }}$, and $V_{\text {th }}$ are the required upper bound for femtocell coverage load, gap, and overlap, respectively, and $L_{i}, G_{i}$, and $V_{i}$ are the current coverage performance observations. $f(s)$ is a function with $L_{\mathrm{th}}, G_{\mathrm{th}}, V_{\mathrm{th}}, L_{i}, G_{i}$, and $V_{i}$ as input parameters. The output parameter is the optimized $\mathrm{CPICH}$ configuration $\mathbf{P}$ which enables the $N$ FBSs to achieve the expected performance.

4.2.3. Genetic Operators. Genetic operators such as crossover and mutation are used to reproduce children from the selected parents. Double point crossover was used in the simulation. Mutation is performed by randomly selecting one of the genes of the chromosome (i.e., pilot power of one

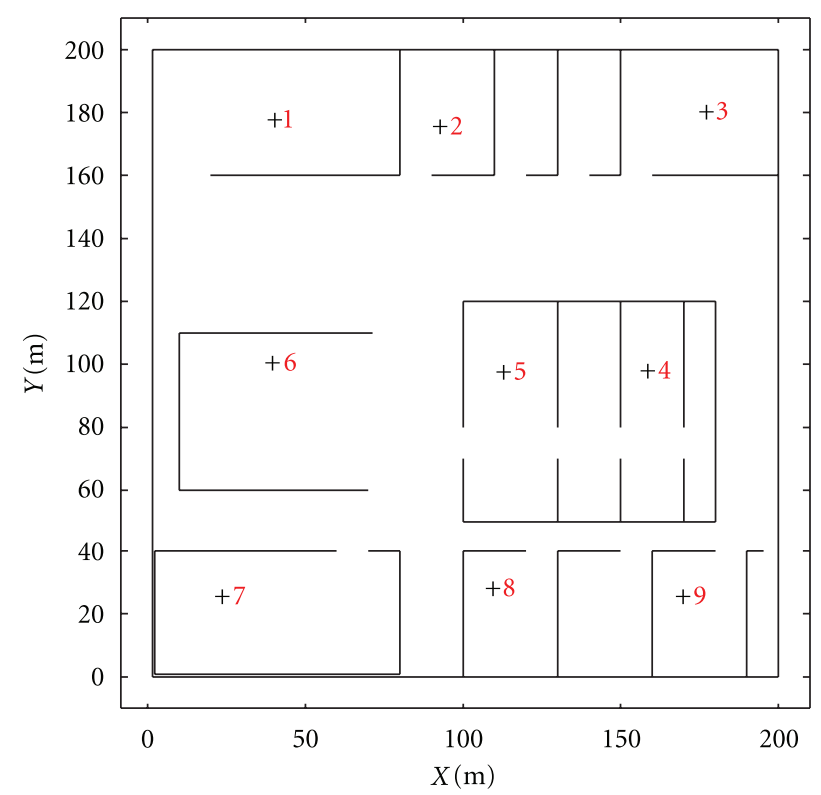

FIgURE 6: Layout of the simulated area.

of the femtocells) then either decrease or increases it by a step size equal to $\Delta \mathfrak{m}$. The decision to increase or decrease the selected femtocell pilot power depends on another random value $\mathfrak{n}$, that is, if $\mathfrak{n}=0$ then this would increase $\mathcal{P}_{l i}$ by $\Delta \mathfrak{m}$, otherwise if $\mathfrak{n}=1$ then $\mathscr{P}_{l i}$ is decreased by $\Delta \mathfrak{m}$.

\section{Simulation Results}

In order to evaluate the effectiveness of the described algorithm, MATLAB was used to simulate the enterprise environment. The simulation scenario is a large typical business area of $200 \mathrm{~m} \times 200 \mathrm{~m}$, it consists of partitioned offices as well as meeting rooms as shown in Figure 6. The partitions are made up of light walls and all other walls are heavy walls. The positions of 9 UMTS femtocells of the $21 \mathrm{dBm}$ class $(125 \mathrm{~mW})$ were assumed to be placed by the enterprise owner without any detailed planning. Their locations can also be found in Figure 6. A number of 400 mobile terminals are uniformly scattered across the entire area. Voice traffic is randomly distributed amongst those mobile terminals such that each of them can generate traffic between 0.2 and 0.5 Erlangs. The value of $\varepsilon$ that is the minimum required signal level for a $\mathrm{FB}$ user equipment (UE) to maintain a $12.2 \mathrm{~kb} / \mathrm{s}$ voice call is assumed to be $-104.18 \mathrm{dBm}$ considering a chip rate equal to $3.84 \mathrm{Mb} / \mathrm{s}$, a $\mathrm{UE}$ noise floor of $-87.50 \mathrm{dBm}$, and a $\mathrm{Eb} / \mathrm{No}$ requirement equal to $8.3 \mathrm{~dB}$ for a voice call as recommended in [32] for additive white Gaussian noise AWGN channel. The path loss model in (4) is used to generate a path loss map for the $200 \mathrm{~m}$ $\times 200 \mathrm{~m}$.

In UMTS systems CPICH is usually set to $10 \%$ of the maximum downlink transmit power. It was found that if the initial pilot power of the FBSs was set to $6 \mathrm{~mW}$ $(7.78 \mathrm{dBm})$ which is half of the maximum pilot power, it would speed up the process. This was done by setting 
TABLe 1: Simulation parameters.

\begin{tabular}{lc}
\hline Simulation parameter & Value \\
\hline Population size $(S)$ & 30 \\
Maximum number of generations & 300 \\
Mutation rate & 0.1 \\
Selection rate & 1 \\
Min femtocell coverage pilot power & $1 \mathrm{~mW}$ \\
Max femtocell coverage pilot power & $12 \mathrm{~mW}$ \\
$W_{1}$ (Attenuation due to light wall) & $3.8 \mathrm{~dB}$ \\
$W_{2}$ (Attenuation due to heavy wall) & $2.7 \mathrm{~dB}$ \\
$L_{\text {th }}$ & $8 \mathrm{Erlangs}$ \\
$G_{\text {th }}$ & 0.1 \\
$V_{\text {th }}$ & 0.3 \\
$\Delta \mathfrak{m}$ & $1 \mathrm{~mW}$ \\
\hline
\end{tabular}

$20 \%$ of the population of the first GA generation to this value and the rest was randomly generated. A roulette wheel selection was performed to select parent solutions for reproduction. Therefore, in this process a parent is selected with a probability that is proportional to its fitness. In order to show the effect of the weighting factor, the weights were changed each time after the entire simulation was executed and then the result was recorded. Other simulation parameters are displayed in Table 1.

Figure 7 illustrates the optimized pilot power coverage when $\omega_{L}=1, \omega_{V}=0.5$, and $\omega_{G}=0.5$. The blue dots represent the scattered mobile terminals. Moreover, Figure 8 shows the load handled by the 9 FBSs. Although the areas covered by the FBS are not equal, yet they are handling very close amount of loads as shown in Figure 6. This is significant in cells 7 and 8 that have the minimum pilot power channel of all other femtocells as in Figure 9 but still handles as much load as the other cells. The fitness for this scenario is 0.8727. It can also be observed in Figure 7 that some areas are not fully covered. This is due to the fact that emphasis has been given to balance the load (i.e., $\omega_{L}=1$ ) for the entire network. Therefore, the OAM does not impose high pilot power for FBS to ensure that the load they are handling does not remarkably deviate from the average.

In the next scenario, the maximum weight is given to minimize the coverage gaps (i.e., maximize the total coverage area). The weights are modified such that $\omega_{L}=0.5, \omega_{V}=$ 0.5 , and $\omega_{G}=1$. The optimized pilot power coverage is presented in Figure 10.

It can be observed that most of the cells in this case increased their pilot power to cover more mobile terminals as illustrated in Figure 12. With those modified weights, it is clear that the overlaps have increased and the load has become unbalanced and some cells become overloaded as shown in Figure 11.

In the third case, the weights have been set as $\omega_{L}=$ $0.5, \omega_{V}=1$, and $\omega_{G}=0.5$. Figure 13 demonstrates the optimized cell coverage where more emphasis is given to minimizing the overlaps. It can be observed that the overlaps have not totally disappeared but instead are minimized as much as possible. This can be seen especially between

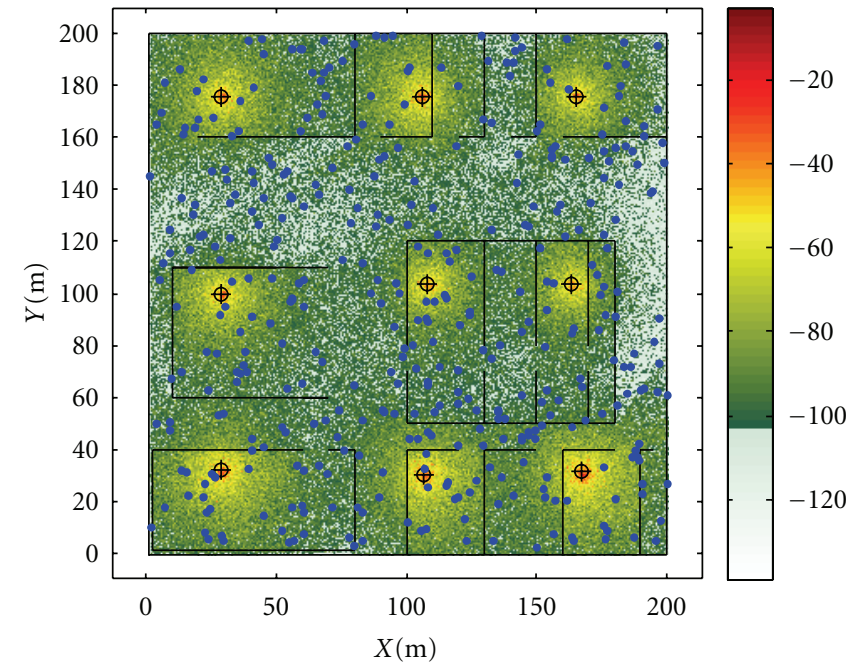

Figure 7: Optimized femtocells coverage when highest weight is given to balance the load, colorbar shows received pilot powers in $\mathrm{dBm}$.

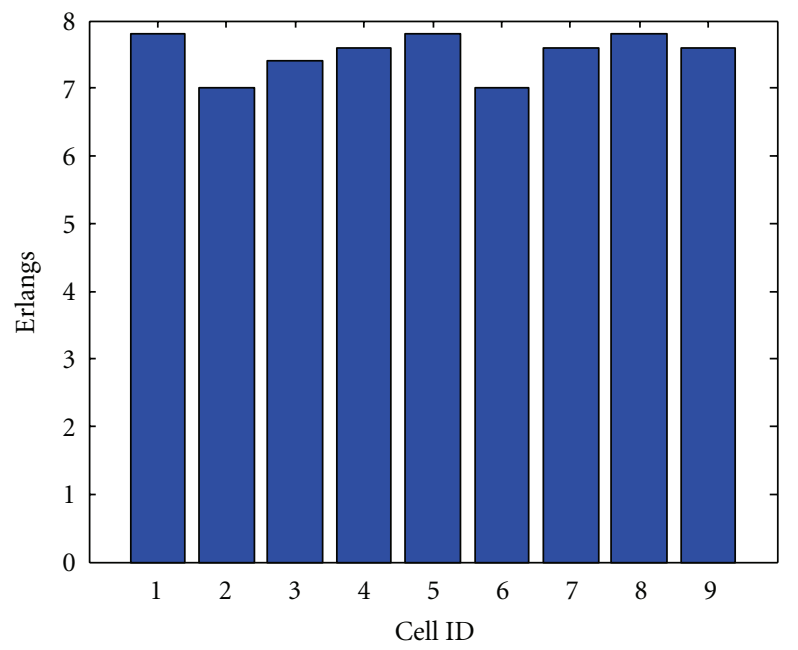

FIgURE 8: Final load handled by the femtocells (no. of FBSs = 9).

cells 2 and 3, and cells 1 and 6 . Controlling the weight given to minimizing the overlaps is important especially in systems that require a certain amount of overlap to perform soft handover from one cell to the other as in CDMA (Code Division Multiple Access). It can also be noticed from Figure 14 that the load distribution across FBSs is not balanced compared to the first case. During the simulation, it was realized that the algorithm decreased the coverage whenever the overlaps increased.

Compared with the simulation results presented in [24] which implements linear programming to minimize the interference and maximize the smallest cell size for femtocells, the proposed algorithm considers load balancing in addition to their objectives. In addition, it performs autonomous optimization of the cell size of a group of femtocells in a more realistic environment where the effect of walls and other obstacles is taken into account when 


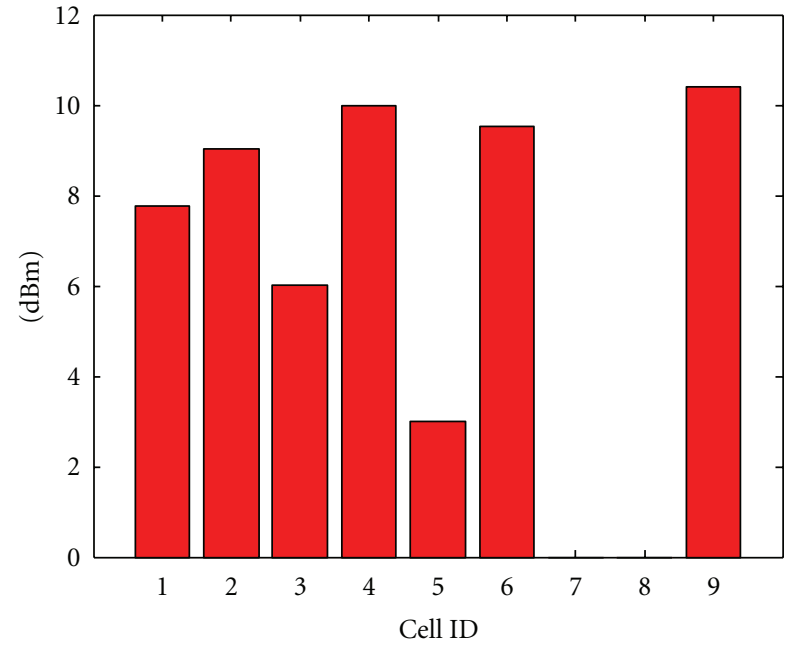

Figure 9: Converged pilot power setting of the femtocells (no. of FBSs $=9$ ).

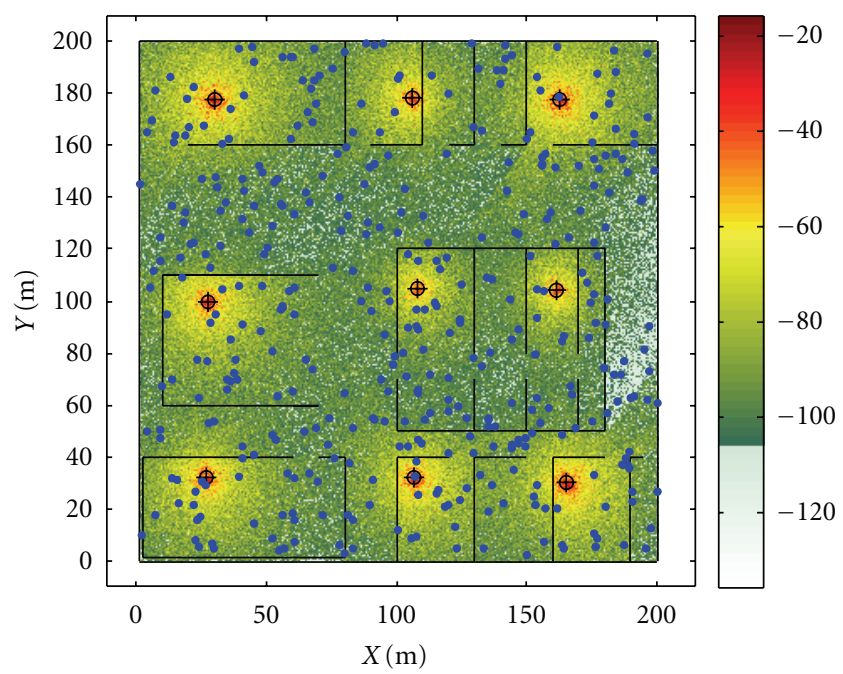

FIGURE 10: Optimized femtocells coverage when highest weight is given to minimize coverage gaps.

estimating the coverage area of the cell. The effect of balancing the load by adjusting the pilot power according to the relative traffic intensity handled in each cell was also considered. Furthermore, unlike the research done in [26], we were able to control the amount of pilot power leakage by altering the weight given to minimizing the overlaps depending on the current traffic distribution.

The results also demonstrated the ability of our centralized algorithm to achieve comparable results to the ones presented in [28] which is based on decentralized genetic programming approach. In addition, it was illustrated that our proposed algorithm is robust to the dynamic changes of the network. That can be evidenced by the short convergence time which is due to the parallelism of the GA. With the roulette wheel selection and reproduction operations, more time will be spent on finding good solutions than moderate ones. Being one of the population-based techniques, GA

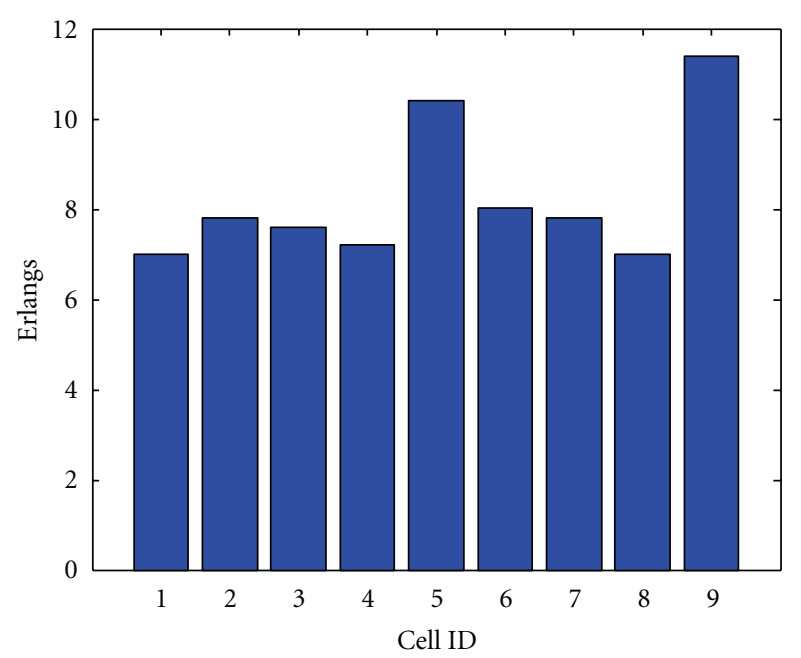

FIgURE 11: Final load handled by the femtocells (no. of FBSs = 9).

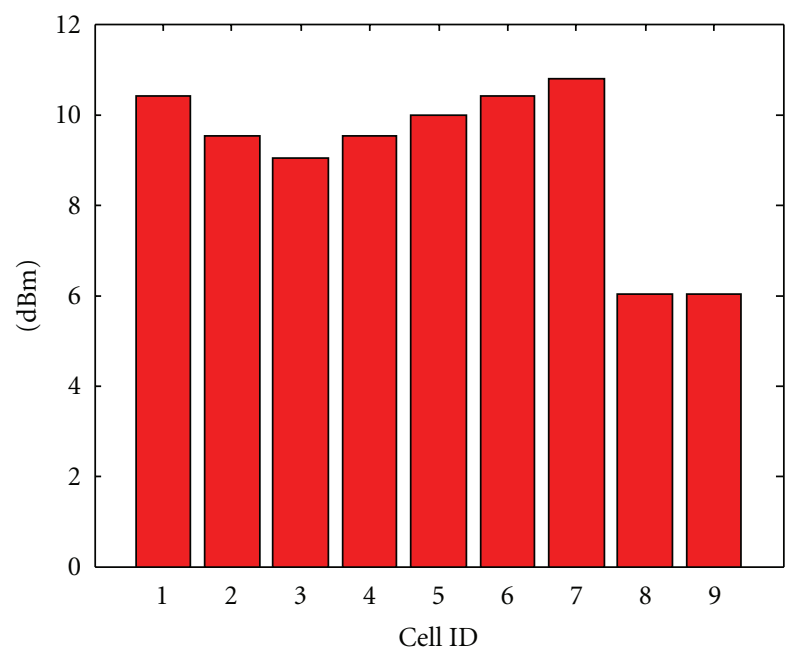

FIGURE 12: Converged pilot power setting of the femtocells (no. of FBSs $=9$ ).

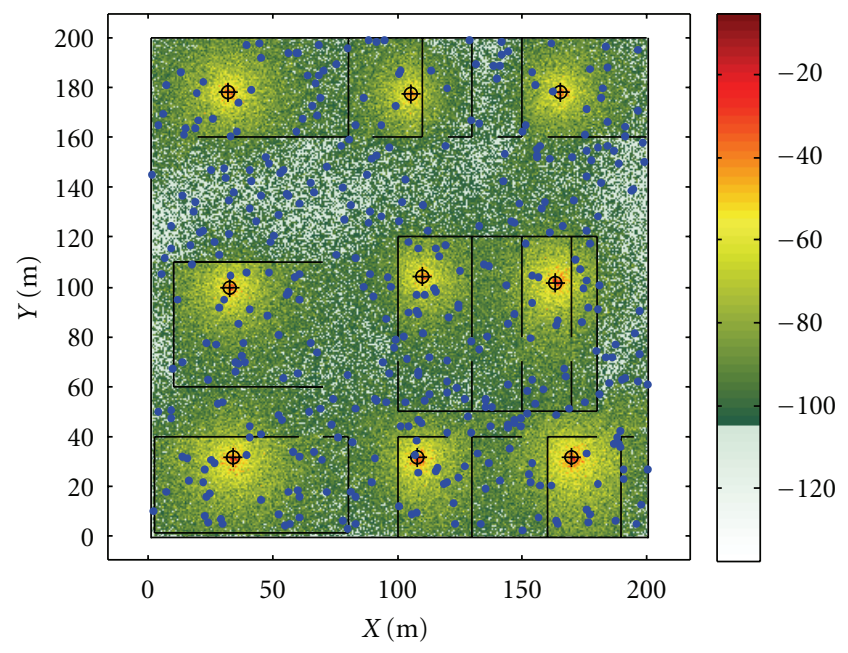

FIGURE 13: Optimized femtocells coverage when highest weight is given to minimize overlaps. 


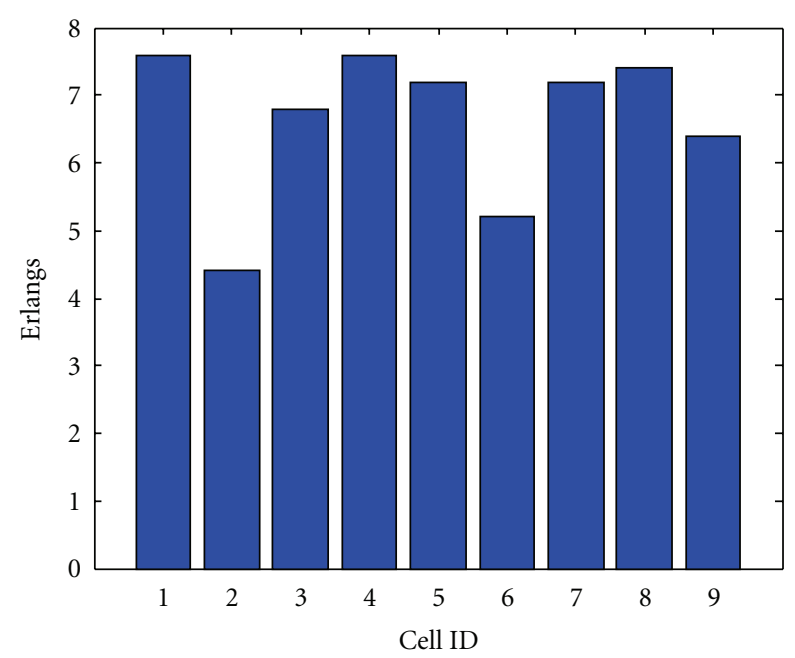

FIgURE 14: Final load handled by the femtocells (no. of FBSs = 9).

TABLE 2: Fixed and optimized power network performance.

\begin{tabular}{lccc}
\hline Mean value & Load & Gaps & Overlaps \\
\hline Fixed power & 0.1667 & 0.2302 & 0.4037 \\
Optimized power & 0.8694 & 0.2749 & 0.1656 \\
\hline
\end{tabular}

provides an opportunity to choose good nonlocal moves from the large space of possibilities.

With the same objective function applied in [28] to a whole network, the authors in [28] presented their results of one scenario with all the weights of (5) set to one whilst our algorithm was evaluated in three different scenarios. A different set of weights was used to emphasize a particular objective. The results were represented in Figures 7, 10, and 13.

The presented approach was also employed to compare with the fixed pilot power scheme mentioned in [18]. In order to do that, the GA was run with $\omega_{V}, \omega_{L}$, and $\omega_{G}$ set to 1 . For the fixed power approach, the pilot power was set to $11 \mathrm{dBm}$ (typically around 1/10th of the total power. Figures 15 and 16 show the load distribution across FBSs when fixed pilot power and optimized pilot power schemes were employed respectively. It can be noticed from Figure 15 that some FBSs are underutilized while others are overloaded. This increases in turn the numbers of dropped and blocked calls. Table 2 demonstrates the differences of network mean load, gaps, and overlaps between the two schemes. The values illustrate the effectiveness of the proposed algorithm to balance the three objectives and to significantly improve the whole network performance.

Table 2 shows that although the mean gaps have increased due to the decreased overlaps, the load is more balanced and pilot power pollution is minimized.

Figure 17 illustrates the final optimized pilot power in this scenario. Compared with the fixed power of $11 \mathrm{dBm}$, the optimized pilot power configuration indicates a $57 \%$ reduction in the total power pollution.

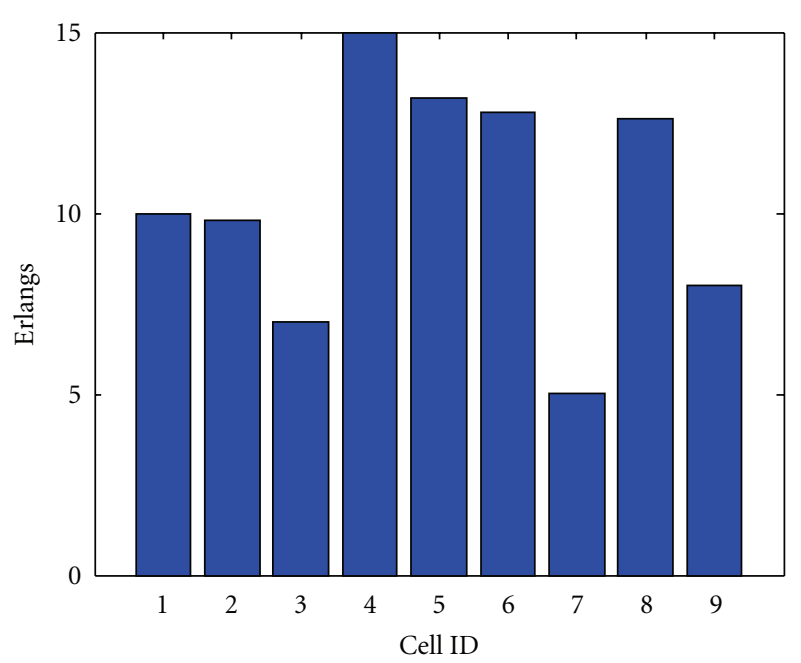

FIGURE 15: Load handled by the femtocells in fixed power (no. of FBSs $=9$ ).

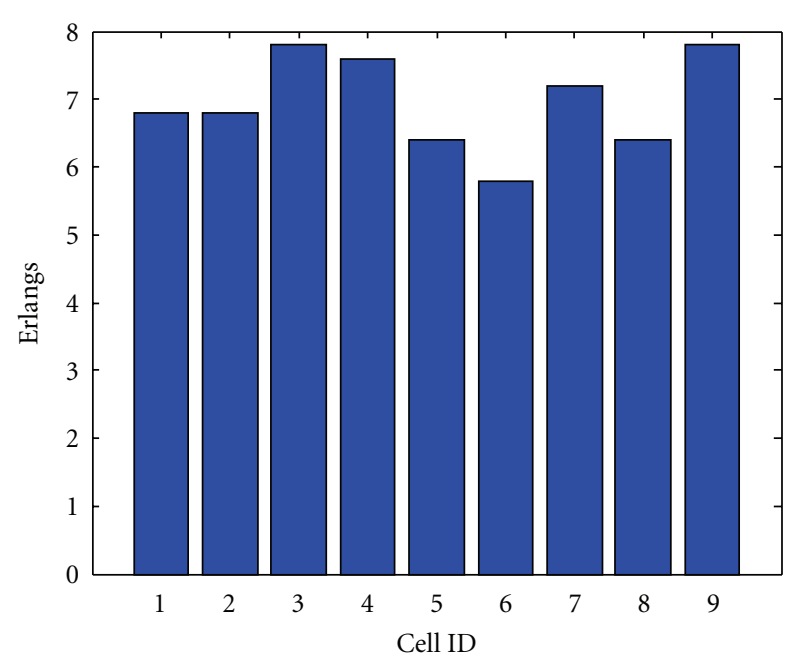

FIGURE 16: Load handled by the femtocells in optimized power (no. of FBSs =9).

Finally, an exhaustive search was performed in order to assess the solution quality of the presented algorithm. In this case, a simplified scenario was considered where only 5 femtocells are deployed. The locations of the FBSs and the UE are identical in both cases in order to precisely compare the performance of GA. Again all weights of the objectives in the fitness function were set to 1 . Figures 18 and 19 demonstrate the final load distribution when executing the exhaustive and the GA, respectively. Similarly, Figures 20 and 21 show the best pilot power configuration as generated from the exhaustive search and the GA, respectively.

It can be seen that the GA is able to produce the optimal pilot power configuration as generated by the exhaustive search. Figure 22 demonstrates the fitness of the solution obtained by the exhaustive search and the converged fitness of the GA. It took $2769 \mathrm{~s}$ for the exhaustive search to find the solution with the highest fitness (0.8797 in this scenario), 


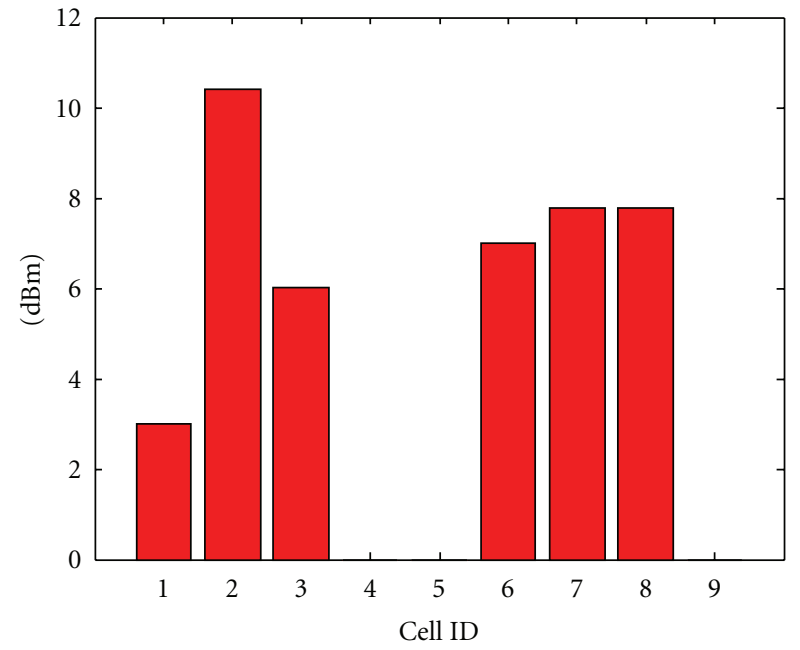

FIGURE 17: Optimized pilot power when all weights set to 1 (no. of FBSs $=9$ ).

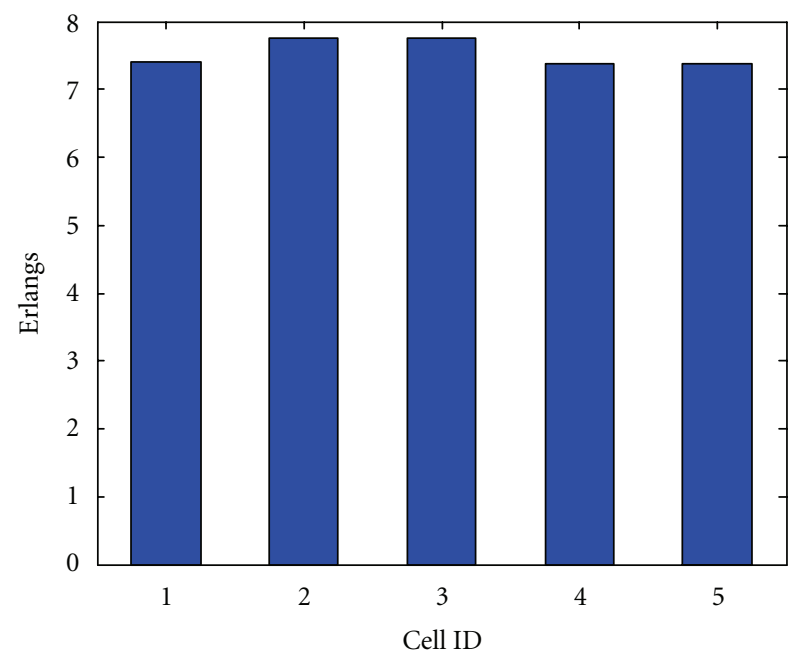

FIGURE 18: Load handled by the femtocells in exhaustive search (no. of FBSs $=5$ ).

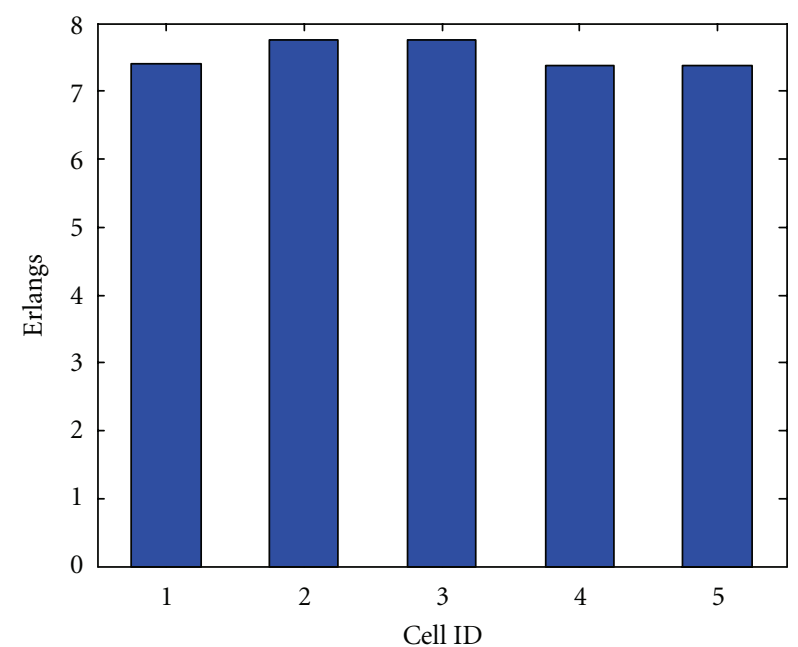

FIGURE 19: Load handled by the femtocells in GA (no. of FBSs = 5).

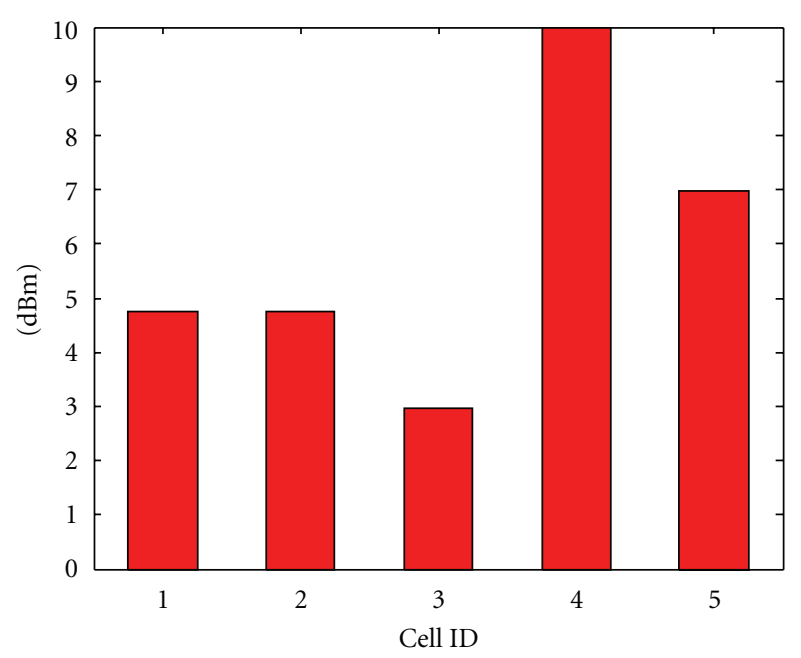

Figure 20: Optimal pilot power given by exhaustive search (no. of FBSs = 5).

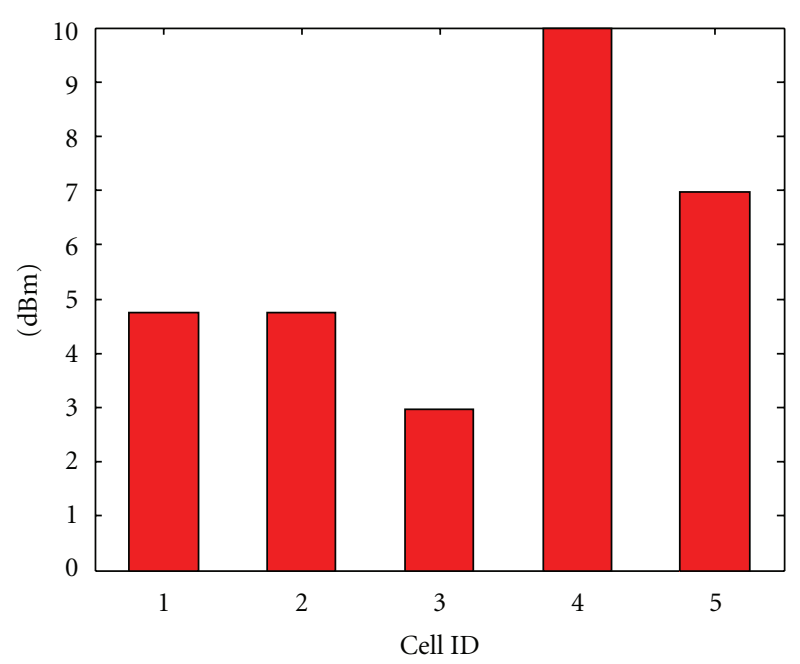

FIGURE 21: Optimized pilot power given by GA (no. of FBSs = 5).

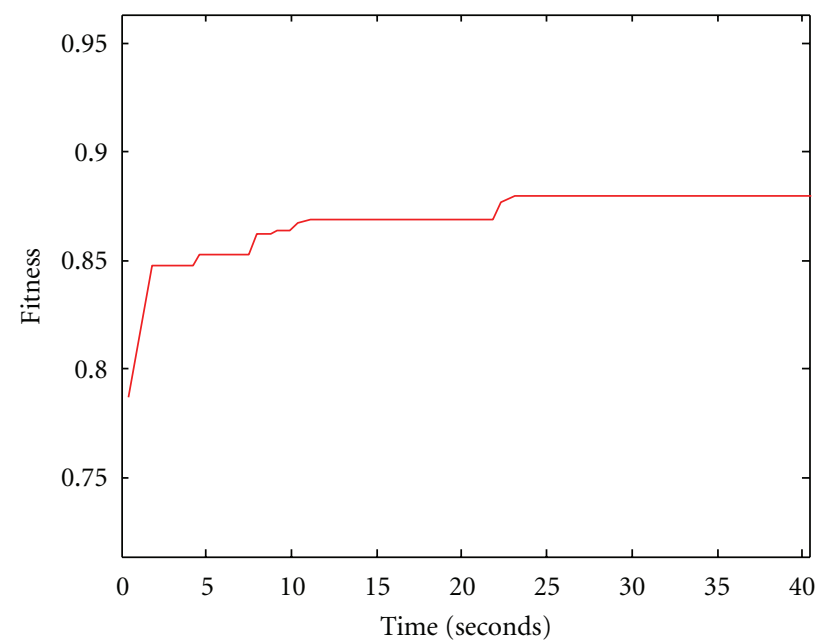

FIGURE 22: GA fitness convergence. 
while it took the GA only $23 \mathrm{~s}$ to produce the same optimal solution. Those results assure that the proposed algorithm can produce a very high quality solution in a fraction of time.

\section{Conclusions \& Future Work}

Self-optimizing capabilities for future mobile network are driven by both the market perspective and the technological potentials. At the same time, femtocells are expected to be widely deployed to enhance indoor radio coverage and system capacity. Their deployment is extending to cover the enterprise area. An approach based on genetic algorithm was presented in this paper to automatically optimize the coverage of a group of femtocells in an enterprise environment. The results demonstrated the ability of the algorithm to dynamically update the pilot powers of the femtocells as per the time varying global traffic distribution and interference levels.

The algorithm was evaluated on UMTS femtocells, but it can work with any air interface by introducing minor modifications. It was also compared with the fixed pilot power scheme and results illustrate a notable network performance and reduction in pilot power pollution. Finally, in comparison with the exhaustive search, the presented algorithm provides an optimal or near optimal solution with significant time reduction.

Future work will include evaluating the effectiveness of the algorithm in a decentralized femtocell deployment. The algorithm will be extended such that it automatically tunes the weights of the objectives of the fitness function depending on the current network requirements. Furthermore, the performance of other optimization methods will be compared to the one proposed in this paper.

\section{References}

[1] Airvana, "Femtocells: transforming the indoor experience," White paper, 2007.

[2] V. Chandrasekhar, J. G. Andrews, and A. Gatherer, "Femtocell networks: a survey," IEEE Communications Magazine, vol. 46, no. 9, pp. 59-67, 2008.

[3] L. Mohjazi, M. Al-Qutayri, H. Barada, K. Poon, and R. Shubair, "Deployment challenges of femtocells in future indoor wireless networks," in Proceedings of the IEEE GCC Conference and Exhibition (GCC '11), pp. 405-408, Dubai, UAE, 2011.

[4] IDATE, "Femtocell worldwide market, Rep," 2010, http://www .idate.org/en/News/Femtocell_650.html.

[5] FemtoForum, http://www.femtoforum.org/femto/.

[6] Informa Telecoms \& Media, "Femtocell Market Status, Rep," 2011, http://www.femtoforum.org/femto/.

[7] G. De La Roche and J. Zhang, "Femtocell networks: perspectives before wide deployments," E-Letter IEEE, 2010.

[8] H. Hu, J. Zhang, X. Zheng, Y. Yang, and P. Wu, "Selfconfiguration and self-optimization for LTE networks," IEEE Communications Magazine, vol. 48, no. 2, pp. 94-100, 2010.

[9] S. Feng and E. Seidel, "Self-organizing networks (SON) in 3GPP long term evolution," Nomor Research GmbH, White Paper, May 2008.
[10] SOCRATES, "Framework for the development of self-organization methods," 2008.

[11] I. Vilovic, N. Burum, and Z. Sipus, "Ant colony approach in optimization of base station position," in Proceedings of the European Conference on Antennas and Propagation (EuCAP '09), pp. 2882-2886, Berlin, Germany, March 2009.

[12] M. Kobayashi, S. Haruyama, R. Kohno, and M. Nakagawa, "Optimal access point placement in simultaneous broadcast system using OFDM for indoor wireless LAN," in Proceedings of the 11th IEEE International Symposium on Personal, Indoor and Mobile Radio Communications (PIMRC '00), pp. 200-204, London, UK, September 2000.

[13] J. Munyaneza, "Optimization of antenna placement in 3G networks using genetic algorithm," in Proceedings of the 3rd International Conference on Broadband Communications, Information Technology and Biomedical Applications, Gauteng, South Africa, 2008.

[14] H. R. Anderson and J. P. McGeehan, "Optimizing microcell base station locations using simulated annealing techniques," in Proceedings of the IEEE 44th Vehicular Technology Conference, pp. 858-862, Stockholm, Sweden, June 1994.

[15] A. Molina, G. E. Athanasiadou, and A. R. Nix, "Automatic location of base-stations for optimized cellular coverage: a new combinatorial approach," in Proceedings of the IEEE 49th Vehicular Technology Conference, pp. 606-610, Houston, Tex, USA, May 1999.

[16] D. Fagen, P. A. Vicharelli, and J. Weitzen, "Automated wireless coverage optimization with controlled overlap," IEEE Transactions on Vehicular Technology, vol. 57, no. 4, pp. 2395-2403, 2008.

[17] I. Siomina et al., "Automated optimization of service coverage and base station antenna configuration in UMTS networks," IEEE Wireless Communications, vol. 13, no. 6, pp. 16-25, 2006.

[18] L. Ho, "Performance of macro- and co-channel femtocells in a hierarchical cell structure," in Proceedings of the 18th Annual IEEE International Symposium on Personal, Indoor and Mobile Radio Communications (PIMRC '07), Athens, Greece, September 2007.

[19] L. T. W. Ho and H. Claussen, "Effects of user-deployed, cochannel femtocells on the call drop probability in a residential scenario," in Proceedings of the 18th Annual IEEE International Symposium on Personal, Indoor and Mobile Radio Communications (PIMRC '07), pp. 1-5, Athens, Greece, September 2007.

[20] H. Claussen, L. T. W. Ho, and L. G. Samuel, "Self-optimization of coverage for femtocell deployments," in Proceedings of the 7th Annual Wireless Telecommunications Symposium (WTS '08), pp. 278-285, Pomona, Calif, USA, April 2008.

[21] D. Lopez-Perez et al., "Intracell handover for interference and handover mitigation in OFDMA two-tier macrocell-femtocell networks," Eurasip Journal on Wireless Communications and Networking, vol. 2010, Article ID 142629, 15 pages, 2010.

[22] G. Guvenc, M.-R. Jeong, F. Watanabe, and H. Inamura, "A hybrid frequency assignment for femtocells and coverage area analysis for co-channel operation," IEEE Communications Letters, vol. 12, no. 12, pp. 880-882, 2008.

[23] V. Chandrasekhar, J. G. Andrews, T. Muharemovic, Z. Shen, and A. Gatherer, "Power control in two-tier femtocell networks," IEEE Transactions on Wireless Communications, vol. 8, no. 8, Article ID 5200991, pp. 4316-4328, 2009.

[24] Y. Y. Li and E. S. Sousa, "Base station pilot management for user-deployed cellular networks," in Proceedings of the IEEE International Conference on Communications (ICC '09), pp. 15, Dresden, Germany, June 2009. 
[25] K. Han et al., "Optimization of femtocell network configuration under interference constraints," in Proceedings of the IEEE 7th International Symposium on Modeling and Optimization in Mobile, Ad Hoc, and Wireless Networks, pp. 1-7, Seol, South Korea, 2009.

[26] Y. Zhang et al., "Pilot power minimization in HSDPA femtocells," in Proceedings of the IEEE Global Telecommunications Conference, Miami, Fla, USA, 2011.

[27] I. Ashraf, H. Claussen, and L. T. W. Ho, "Distributed radio coverage optimization in enterprise femtocell networks," in 2010 IEEE International Conference on Communications (ICC '10), Cape Town, South Africa, May 2010.

[28] L. T. W. Ho, I. Ashraf, and H. Claussen, "Evolving femtocell coverage optimization algorithms using genetic programming," in Proceedings of the IEEE 20th Personal, Indoor and Mobile Radio Communications Symposium (PIMRC '09), pp. 2132-2136, Tokyo, Japan, September 2009.

[29] Z. Wei, Z. Feng, Y. Li, and Q. Zhang, "Voronoi-based coverage optimization for multi-femtocells," in Proceedings of the IEEE International Conference on Wireless Information Technology and Systems (ICWITS '10), Honolulu, Hawaii, USA, September 2010.

[30] S. M. Sait and H. Youssef, Iterative Computer Algorithms with Applications in Engineering, IEEE Computer Society, Calif, USA, 1999.

[31] S. N. Sivanandam and S. N. Deepa, Introduction to Genetic Algorithms, Springer, Berlin, Germany, 2008.

[32] 3GPP Technical Specification 25.104 v.7.9.0, "Base Station (BS) radio transmission and reception (FDD)," 2008, http://3gpp.org/. 

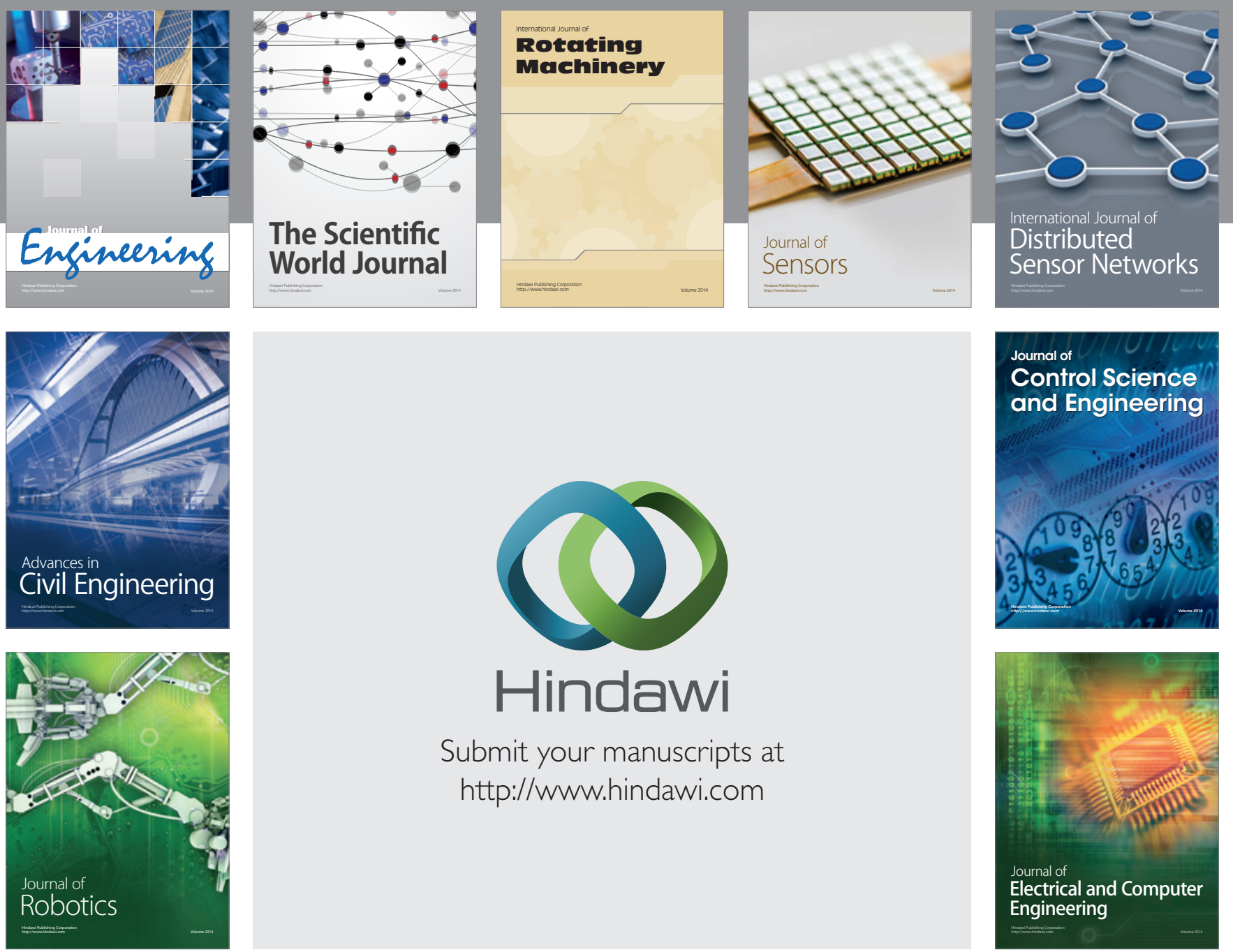

Submit your manuscripts at

http://www.hindawi.com
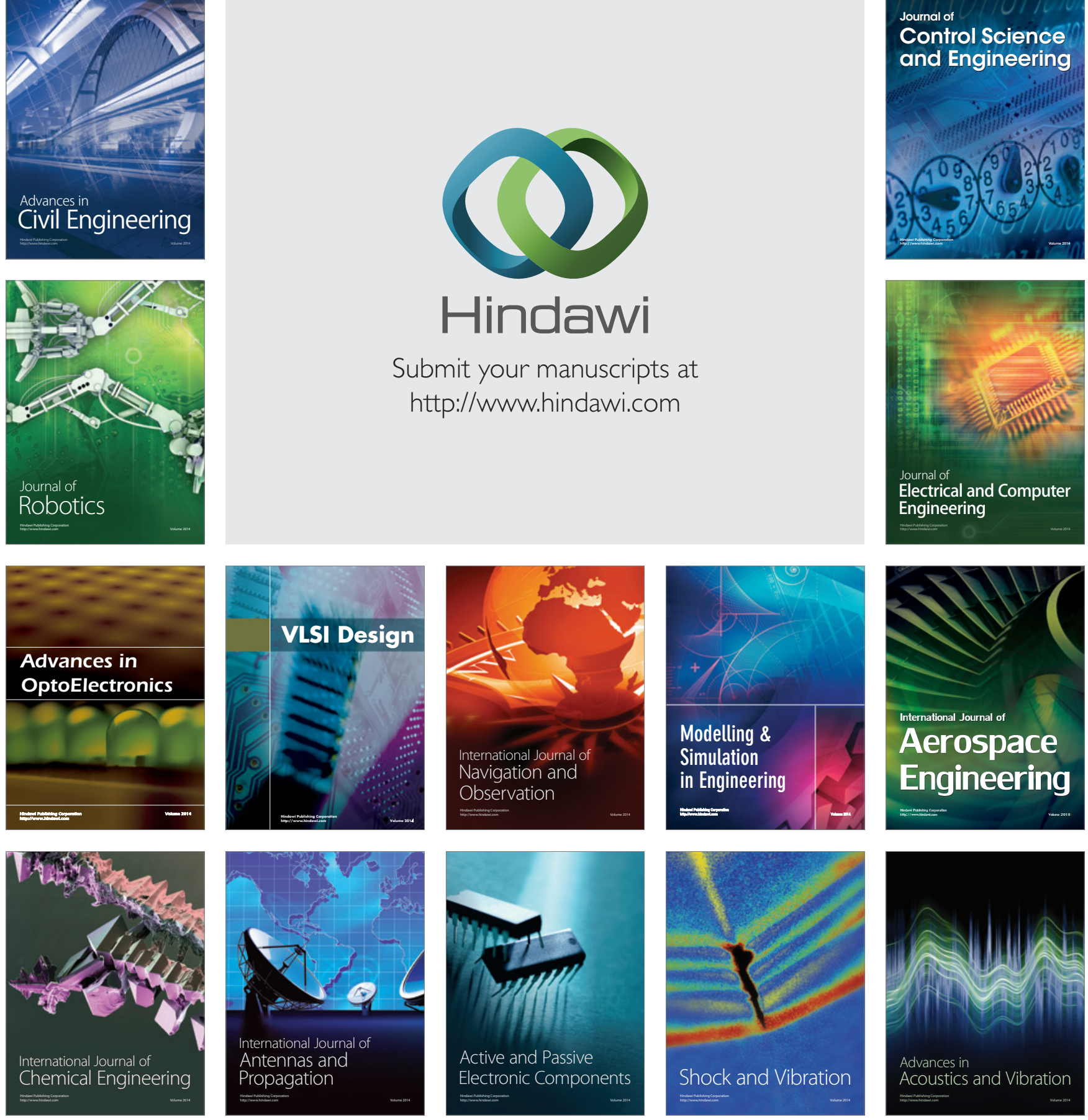